\title{
Der Thesaurusgedanke im Grimmschen Wörterbuch
}

\author{
Michael Schlaefer, Deutsches Wörterbuch, \\ Akademie der Wissenschaften zu Göttingen, Deutschland
}

\begin{abstract}
The Thesaurus Concept in Grimm's German Dictionary. Users often expect to find a complete collection of German vocabulary and quotations in Grimm's German Dictionary. These opinions result from different pretensions of Grimm lexicographers to produce a thesaurus. A historical analysis allows us to discern four different thesaurus concepts in the development of the German Dictionary. During the first period the concept was determined by the attempts of J. Grimm to document the richness of the entire German language in only one dictionary. With regard to certain puristic selections this thesaurus concept can be described as a 'regulated selected richness'. In the following period regulating elements were replaced by philological scientific methods to give reasons for the maintenance of what $R$. Hildebrand called internal completeness. The new scientific argument of completeness is the basis for the rise of the German Dictionary to the state of a national thesaurus as well as the slowing down of production. Due to this the dictionary lost part of its acceptance, and the planning of an alternative thesaurus was started. When, in 1908, the Prussian Academy took charge of the German Dictionary the third period of the thesaurus concept, which included several reforms, began. Above all, the claim for completeness was limited to the variety of cultivated speech. These reforms, however, could only solve part of the problems. It was impossible to produce complete articles on philological standards and to finish the dictionary in an adequate time. With the new edition of the German Dictionary, started in 1957, these problems continued. Since 1987 the office in Göttingen tries to go new ways by separating the functions of a dictionary from the functions of a thesaurus. This fourth concept is based on a functional separation between selective dictionary articles and a complementary data-based register of texts.
\end{abstract}

Keywords: ACADEMY DICTIONARIES, COLLECTION OF QUOTATIONS, CONCEPTION, DICTIONARY, DICTIONARY ENTRIES, DICTIONARY PLANNING, DICTIONARY STYLES, DICTIONARY USERS, GRIMM'S DICTIONARY, HISTORY OF DICTIONARIES, THESAURUS

Zusammenfassung: Benutzer betrachten das Grimmsche Wörterbuch vielfach als vollständige Sammlung aller deutschen Wörter und ihrer Belegstellen. Diese Auffassung ist auf verschiedene Ansprüche von Grimm-Lexikographen zuruckzuführen, einen Thesaurus zu erstellen. Unternehmensgeschichtlich ist es möglich, vier verschiedene Thesauruskonzepte in der Entwicklung des Deutschen Wörterbuches zu unterscheiden. In der ersten Periode wurde das Thesauruskonzept vom Bemühen J. Grimms bestimmt, die ganze Fülle der deutschen Sprache in nur einem Wörterbuch zu erschließen. Da er nach bestimmten individuellen Gesichtspunkten auswählt, kann vom Thesauruskonzept der setzend gewichteten Fülle gesprochen werden. In der zweiten Periode 
wurden die setzenden Elemente durch philologisch-wissenschaftliche Vollständigkeitsbegründungen abgelöst. Die neue wissenschaftliche Begründung des Vollständigkeitsanspruchs, die $R$. Hildebrand als innere Vollständigkeit umschreibt, schafft die Grundlage dafür, daß das Grimmsche Wörterbuch zum Nationalthesaurus wird, führt aber auch zu einer erheblichen Verlangsamung der Produktion. Daher entstehen Akzeptanzverluste und es kommt zu Planungen für einen Konkurrenz-Thesaurus. Mit der Übernahme durch die Preußische Akademie werden wichtige Reformen beim Deutschen Wörterbuch eingeleitet. Sie markieren den Beginn der dritten Phase des Thesauruskonzepts. Vor allem kommt es zu einer Beschränkung des Vollständigkeitsanspruchs auf die Schriftsprache. Die bestehenden Probleme konnten durch die Reformen jedoch nur zu einem Teil gelöst werden. Philologisch verstandene Vollständigkeit in den Wörterbuchartikeln war nicht mit dem Bemühen um einen Abschluß des Wörterbuchs in vertretbarer Zeit zu verbinden. Die Neubearbeitung des Deutschen Wörterbuchs führte diese Probleme fort. Die Göttinger Arbeitsstelle versucht seit 1987, mit einem neuen Thesauruskonzept zu einer Lösung zu kommen. Dieses vierte Konzept beruht auf einer funktionalen Trennung von selektiven Wörterbuchartikeln und einem komplementären datenbankgestützten Großarchiv.

Stichwörter: AKADEMIEWÖRTERBÜCHER, BELEGARCHIVE, DARSTELLUNGSSTILE, GRIMMSCHES WÖRTERBUCH, KONZEPTION, STICHWORTBESTAND, THESAURUS, WÖRTERBUCH，WÖRTERBUCHBENUTZUNG，WÖRTERBUCHGESCHICHTE，WÖRTERBUCHPLANUNG

Unter den Anfragen, die von Benutzern an die Göttinger Arbeitsstelle des Deutschen Wörterbuchs gerichtet wurden, fand sich kürzlich die Bitte eines Wissenschaftlers, man möchte zwei nicht näher zugeordnete Textstellen aus der jüngeren Literatur nach Autor und Werk identifizieren. Offensichtlich ging dieser Benutzer davon aus, daß die Arbeitsstellen des Grimmschen Wörterbuchs über vollständige Belegarchive für die deutsche Literatursprache verfügen, in denen analog zum Thesaurus Linguae Latinae jede beliebige Textstelle aus der Literatur mit geringem Aufwand ermittelbar sei. So wie dieser Benutzer die Vollständigkeit des Belegmaterials in den Archiven des Deutschen Wörterbuchs erwartete, wird aus weiteren Anfragen erkennbar, daß andere Benutzer auch von der Vollständigkeit der Wortschatz- und Belegabbildung in den Wörterbuchartikeln überzeugt sind. Kennzeichnend für diese Einstellung ist zum Beispiel die verärgerte Beschwerde eines Benutzers darüber, daß neben dem Stichwort DROSSELBART auch andere Komposita in der Neubearbeitung des Grimmschen Wörterbuchs fehlten. Der Versuch, die Notwendigkeit zur Auswahl auch in einem lexikographischen Großprojekt zu erläutern, überzeugte diesen Benutzer nicht. Früher habe im Grimmschen Wörterbuch alles gestanden und jeder Artikel sei ein Maßanzug gewesen, heute habe man es wohl nur noch mit Stangenware zu tun, lautete sein Kommentar. Die Benutzererwartung, im Grimmschen Wörterbuch müsse "alles" stehen, wurde auch auf einer im Herbst 1993 in Wien veranstalteten Arbeitstagung deutschsprachiger Akademiewörterbücher vertreten (Tätigkeitsbericht 1993/94: 18-21). Hinter solchen Erwartungen steht die Identifikation des 
Grimmschen Wörterbuchs mit einem Thesaurus Linguae Germanicae, also einem Wörterbuch, das die deutsche Sprache vollständig erfaßt, beschreibt und in Belegen verfügbar macht. Die Realitätsferne dieser Erwartung läßt sich leicht erkennen, wenn man die Quellen- und Beleglage für die abgeschlossene Ausgabe des Grimmschen Wörterbuchs kennt. Die etwa 25.000 Titel, die das Quellenverzeichnis zum Grimmschen Wörterbuch verzeichnet, repräsentieren nur einen Bruchteil der insgesamt geschichtlich überlieferten deutschen Texte, und das Belegmaterial, das aus diesen Texten gewonnen wurde, stellt nur einen kleinen Teil der insgesamt enthaltenen Wortvorkommen dar. Entsprechendes gilt auch für die Neubearbeitung des Deutschen Wörterbuchs, dessen Göttinger Teil auf einer Materialbasis von etwa 6000 Quellen und rund 2,5 Millionen Belegen nur für die Buchstaben D-F beruht. Kritiker des Grimmschen Wörterbuchs werden so leicht immer wieder Lücken im Stichwortbestand, in der Beschreibung einzelner Wortverwendungen und in der Belegabbildung finden (z. B. Sanders 1852).

Der Widerspruch zwischen der Benutzererwartung, eine vollständige lexikographische Kodifikation der deutschen Lexik im Grimmschen Wörterbuch $\mathrm{zu}$ finden und der offensichtlich traditionell selektiven Ausrichtung dieses Wörterbuchs führt auf die Frage nach der Entstehung der Vollständigkeitsvorstellung. Anhand einer kurzen geschichtlichen Skizze soll versucht werden, die Ursachen und die Entwicklung verschiedener Thesauruskonzepte in den Bearbeitungsabschnitten des Wörterbuchs zu verfolgen.

Als Jacob und Wilhelm Grimm mit den Vorarbeiten für das deutsche Wörterbuch 1838 begannen, stand der Vollständigkeitsgesichtspunkt nicht im Mittelpunkt der konzeptionellen Planungen, er klang jedoch deutlich an. Der Begriff der Vollständigkeit findet sich in der Einleitung zum ersten Band im Zusammenhang mit der Beschreibung des Zwecks, zu dem das Wörterbuch entstehen sollte. Der "ganze schatz" der deutschen Sprache sollte mit dem Deutschen Wörterbuch bewahrt werden, heißt es dort (DWB I 1854: XII).

Liest man weiter, steht dieser Totalitätsanspruch in unvermitteltem Widerspruch zu dem explizit angekündigten Verzicht auf die Aufnahme systematischer Ableitungen und Komposita (DWB I 1854: XLII, XXV, XLV). Läßt man sich jedoch auf Jacob Grimms nähere Erläuterungen zur Begrenzung der Stichwortaufnahme ein, wird deutlich, daß im Verständnis des Begriffs Schatz die Lösung des Widerspruchs liegt. Die systematischen Wortbildungsreihen zeugen für Jacob Grimm nicht vom Reichtum der Sprache, "blosz von dem zwang, der ihrer syntax angethan wird" (DWB I 1854: XXV). Der "ganze schatz" bezeichnet daher keine absolut vollständige Sammlung aller überhaupt bezeugten Wörter des Deutschen, sondern einen spezifischen Teilausschnitt, in dem der "gehalt und das innerste der sprache am klarsten zu tage kommt" (Kirkness 1980: 272). Neben den Simplizia sind dies, wie die Betrachtung des Wörterbuchs zeigt, vor allem Wörter, die den Vorstellungen der Grimms von Poetizität, sprachnationaler Ursprünglichkeit und genetisch "richtiger" Sprachentwicklung entsprechen. 
Im Blick auf den hier behandelten Vollständigkeitsbegriff kann man in der Gründungsphase des Unternehmens von einer gewichteten Fülle als dem maßgeblichen Prinzip sprechen. Diesem Prinzip entsprechend fand in erkennbarem Umfang auch veralteter Wortschatz Aufnahme, wurden mundartliche, umgangssprachliche und sondersprachliche Wörter und Verwendungsweisen einbezogen. Die Vision Jacob Grimms von seinem wie ein Füllhorn zu benutzendes Wörterbuch, das Hausbuch der Deutschen werden sollte (DWB I 1854: XII-XII), läßt ganz deutlich erkennen, wie stark die Überzeugung wirkte, mit diesem Werk eine unerschöpfliche Fundgrube, einen für alle Zwecke ausreichenden Wortvorrat geschaffen zu haben.

Die Wörter Schatz und Wortvorrat führen leicht auf das lateinische Wort thesaurus, das im außerterminologischen Gebrauch unter anderem auch die Bedeutungen 'reicher Vorrat', 'Hülle und Fülle' oder 'Fundgrube' zeigt (Georges 1951). Die frühe Ausrichtung des Deutschen Wörterbuchs an einem Thesauruskonzept läßt sich durch weitere Beobachtungen stützen. Die Darstellung der Fülle, des geschichtlichen Reichtums des Deutschen, so ist zunächst zu erkennen, bildete ein so dominantes Konzeptionselement, daß ihr gegenüber strenge lexikographische Strukturierungen zurückgenommen wurden. Damit erklärt sich teilweise die auffällige Ungegliedertheit der Wörterbuchartikel in bezug auf die geschichtlichen Zusammenhänge und sprachlichen Schichten (vgl. Stötzel 1970: 14-16).

Den Stichwortreihen, den Einzelwörtern und den Belegen kommt im Konzept Jacob Grimms weniger die Aufgabe zu, strukturelle oder wortgeschichtliche Schichtungen und Gliederungen der Lexik zu verdeutlichen. Sie sollen vielmehr den Benutzern als Teile einer reichen, wertvollen, gemeinsamen Tradition präsentiert werden. So soll Jacob Grimm in seiner Göttinger Antrittsvorlesung geäußert haben, in nichts anderem zeige sich das Band der Vaterlandsliebe stärker, als in der Gemeinsamkeit der Sprache (Pfütze 1962: 266). Der Sprachnation sollte folglich das Deutsche Wörterbuch als ein Heiligtum gewidmet werden (DWB I 1854: XII). Solche Formulierungen stellen sich sehr deutlich neben die zum lateinischen thesaurus gehörende Bedeutung 'Schatzkammer eines Tempels' und weisen zudem in die Wörterbuchgeschichte und Projekte der barocken Sprachmeister zurück.

Die Diskussion darüber, ob sich Jacob Grimm als "Sprachmeister" (Schirokauer 1957: 318) oder Hüter des nationalen Wörterbuchheiligtums verstand, kann hier nur am Rand verfolgt werden. Der Entrüstung eines Teils der Grimm-Literatur, in der solche Tendenzen ausgeschlossen werden (u. a. Dückert 1987: 12), ist die Fülle an normativen Zusätzen, Auswahlgesichtspunkten und wertenden Eingriffen entgegenzustellen, die sich in den frühen Wörterbuchteilen findet. Vor allem aber spricht für die Annahme einer selbstgewählten Sprachmeisterrolle die nachdrückliche Berufung Jacob Grimms auf das italienische Crusca-Wörterbuch (Kirkness 1980: 74), die zugleich auch die Brücke zu den angedeuteten barocken, sprachmeisterlichen Thesaurus-Aspekten schlägt. 
Das von der Academia della Crusca im 17. Jahrhundert herausgegebene Wörterbuch stellte in normativer Form die "vorbildliche" toscanische Varietät lexikographisch dar. Das Grimmsche Wörterbuch sollte wohl, wie die weiten Ausgriffe in volks- und umgangssprachliche Schichten zeigen, kein "Gesetzbuch" (Schirokauer 1957: 318) der deutschen Sprache werden, aber ein Wörterbuch, das im unmittelbaren Anschluß an das Vorbild des Crusca-Wörterbuchs setzend und weniger in einem wissenschaftlichen Verständnis systematisch den richtigen Ausschnitt aus der Fülle zeigte.

Die Berufung auf das Crusca-Wörterbuch stellt den Grimmschen Ansatz zugleich in die Tradition der Wörterbuchplanung seit dem 17. Jahrhundert. $\mathrm{Ph}$. Harsdörfer hatte vorgeschlagen, aus dem Kreis der Fruchtbringenden Gesellschaft ein vollständiges Wörterbuch hervorgehen zu lassen. Als Vorbild dafür galt eben das Crusca-Wörterbuch (DWB V 1873: III-IV). Dieser nicht verwirklichte Plan findet sich in modifizierter Form bei G. W. Leibniz. Anstelle eines einzigen vollständigen deutschen Wörterbuchs wollte G. W. Leibniz allerdings eine Anzahl funktionell differenzierter Wörterbücher in der Berliner Akademie erarbeiten lassen (Leibniz 1717: 44 ff). Vollständigkeit und das Ziel "Reichtum, Reinigkeit und Glanz" (Leibniz 1717: 64) des Deutschen lexikographisch zu erschließen, bilden die gemeinsamen Merkmale der genannten Wörterbuchprogramme und des Grimmschen Wörterbuchs. Das Grimmsche Wörterbuch folgt eng dem Harsdörferschen Konzept von dem einen vollständigen Gesamtwörterbuch. Wenn Jacob Grimm im Vorwort zum Deutschen Wörterbuch von der günstigen Zeit (DWB I 1854: III) spricht, in der sein Vorhaben zu verwirklichen sei, läßt sich dies nur auf die vorausgehende Tradition beziehen, in der wissenschaftlich und organisatorisch ein solches Unternehmen ausgeblieben war.

Noch deutlicher wird das Grimmsche Traditionsverständnis, wenn beklagt wird, daß das Deutsche Wörterbuch nur mangelhaft wissenschaftsorganisatorisch eingebettet sei (Kirkness 1980: 69). Es fehlt ihm eben die für die älteren Wörterbuchprojekte fest eingeplante Anbindung an eine maßgebliche akademische Einrichtung oder gar einen nationalen Träger.

Die Komponenten 'Fülle' und 'nationalsprachliches Gesamtwerk des Deutschen' wurden für das Grimmsche Wörterbuch auch in der Generation, die den Grimms in der Bearbeitung folgte, beibehalten, teilweise sogar noch verstärkt. Es traten jedoch hinsichtlich der Vollständigkeitsansprüche deutliche Veränderungen ein. Am Beispiel der von R. Hildebrand bearbeiteten Teile des Wörterbuchs sollen im weiteren kurz die sich daraus ergebenden Modifikationen des Thesauruskonzepts erläutert werden.

In seiner Antrittsvorlesung in Leipzig betonte R. Hildebrand 1869 die doppelte Bedeutung des Deutschen Wörterbuchs als nationale und wissenschaftliche Aufgabe (Hildebrand 1869: 3). Das Grimmsche Wörterbuch umschrieb er als "schatzhaus des deutschen geistes" (Hildebrand 1869: 7), in dem die Besitztümer des Volkes zu einem Spiegel seiner Lebensvielfalt entwickelt wurden. "Unser wörterbuch wird so von selbst zugleich zu einem buche deutscher 
geschichte, denn mit und in den wörtern zieht das leben der nation, das innere und äuszere an uns vorüber. (...). Das wörterbuch arbeitet zugleich (...) an einer deutschen, im gewissen sinne europäischen culturgeschichte, die die königin der wissenschaften zu werden sich anschickt" (Hildebrand 1869: 10). In diesem Verständnis war das Grimmsche Wörterbuch das zentrale Instrument nationaler Geschichtsschreibung, in dem nicht nur Sprachgeschichte ihren Niederschlag fand, sondern vor allem Begriffs-, Kultur- und Sachgeschichte über sprachliche Quellen erschlossen wurden. An einer anderen Stelle desselben Referats findet sich der Begriff des "nationalschatzes", der sogar von so hohem Wert sein könne, daß selbst "ein schon vergehendes volk durch die wiedergeburt der sprache neu zu bilden" sei (Hildebrand 1869: 12).

Die Gleichsetzung von Nationalthesaurus und Grimmschem Wörterbuch ist überdeutlich zu erkennen. Mit der zwei Jahre nach R. Hildebrands Rede vollzogenen Reichsgründung gewann die nationale Anbindung des Unternehmens auch eine konkrete politische Qualität. War das Grimmsche Wörterbuch seit seiner Gründung eine Art fachlicher Kristallisationspunkt der wissenschaftlichen Germanistik gewesen, so läßt die nicht unwesentliche staatliche Förderung ab 1868 darauf schließen, daß die politisch Verantwortlichen die Einschätzung des Werks als "Nationalschatz" teilten. Eine solche Anerkennung bedeutete eine wesentliche wissenschaftspolitische Aufwertung des Wörterbuchs, auch wenn sich dies zunächst nicht in einer konkreten äußeren Institutionalisierung niederschlug.

Die wissenschaftspolitische Aufwertung des Grimmschen Wörterbuchs zum Unternehmen von nationalem Interesse ist ein wesentliches Kennzeichen der zweiten Phase der Entwicklung des projekteigenen Thesauruskonzepts. Wohl im wesentlichen von dieser Entwicklung her ist die Veränderung des ursprünglichen Fülle-Begriffs als determinierendes Thesaurusmerkmal zu erklären. Als wissenschaftliches Projekt von nationalem Interesse stand das Werk auch exemplarisch für die wissenschaftlichen Standards. Ein eher individuell gesetzter Fülle-Umriß war mit einem solchen Anspruch nicht zu vertreten. Schon die erwähnte Erweiterung des Objektbereichs um die Erfassung kultur- und begriffsgeschichtlicher Zusammenhänge bedeutete gegenüber dem ursprünglichen Wörterbuchziel einen beträchtlichen Zuwachs an Umfang. Es wurde jedoch vor allem das Bemühen wirksam, Vollständigkeit auf einer ganz anderen Ebene zu erreichen, als es die Unternehmensgründer versucht hatten.

In bezug auf Kritik an Lücken im Stichwortbestand und den Wortbezeugungen erläutert $R$. Hildebrand im Vorwort zu Band V, daß die äußere Vollständigkeit in Form der Buchung aller jemals bezeugten Wörter wohl nicht zu erreichen sei. Es gehe vielmehr um die Verwirklichung der "inneren Vollständigkeit" (DWB V 1873: IX). Diese Formel von der inneren Vollständigkeit kann man auf die für wissenschaftliche Arbeit grundsätzlich zu fordernde Intersubjektivierbarkeit, Systematik und inhaltliche Ausschöpfung des Themas beziehen, dire zwar nicht im statistischen Sinne jedes Einzelvorkommen erfaßt, 
wohl aber differenzierend und problematisierend ein in den Zusammenhängen umfassendes, vollständiges Verständnis gewährleistet, wie es für Monographien üblich ist. Das Konzept der setzend gewichteten Fülle wurde so durch ein Konzept der wissenschaftlich begründeten Vollständigkeit abgelöst. Damit verschwanden dem wissenschaftlichen Verständnis der Zeit entsprechend normative Tendenzen zugunsten einer stärker deskriptiven Beschreibungshaltung. Das neue Konzept der wissenschaftlichen Vollständigkeit führte jedoch alsbald zu einer Reihe schwerwiegender Probleme. Vor allem zeigte sich, daß der neue wissenschaftliche Vollständigkeitsbegriff zwar in den einzelnen Artikeln weiterführte, daß aber die Bindung an verschiedene Erkenntnisinteressen und die stark auf das Einzelwort fixierten Ansätze eine mangelhafte Vergleichbarkeit verschiedener Artikel bewirkten.

Die Materialgrundlage von ca. 600.000 Belegen für den gesamten Wortschatz, die zu Beginn der Arbeiten am Deutschen Wörterbuch gesammelt worden war (Dückert 1987: 34), reichte für das erweiterte Thesauruskonzept keinesfalls aus. Wollte man im philologischen Verständnis über die Sprache in die Kultur- und Geisteswelt vordringen und Wortgeschichte wissenschaftlich vollständig darstellen, waren erheblich dichtere Materialmengen gefordert. Es gehörte folglich zum Repertoire aller Grimm-Bearbeiter aus dem letzten Viertel des 19. Jahrhunderts, über eine mangelhafte Beleglage zu klagen, die durch zeitaufwendige Sammlungen nachgebessert werden mußte (DWB VI 1885: I). Treffend hat R. Meißner diesen Zustand mit dem Vergleich vom Haus ohne Fundament (Meißner 1910: 76) charakterisiert. Die Komplexität des Objektbereichs und der Anspruch der inneren Vollständigkeit führten zudem in den Artikeln zu einer wesentlich breiteren Belegabbildung. Sie machten auch explizite Erklärungen in weit größerem Maß notwendig, als es bis dahin üblich gewesen war. Daraus entwickelte sich folgerichtig ein Artikelaufbau in Form der Kurzmonographie, der von den Zeitgenossen als Übergang zum "großen stil" (DWB VII 1889: I) empfunden wurde. In der Folge dieser Veränderungen und der Ausrichtung der. Artikelarbeit an verschiedenen wissenschaftlichen Erkenntnisinteressen nahm die Eigenwilligkeit der einzelnen Lexikographen in einem Maß zu, das für ein Wörterbuch unverträglich war. Die boshaften Anmerkungen A. Hübners zum Deutschen Wörterbuch als einem "Rumpf mit vielen Gliedern, aber ohne Kopf" oder vom "diadochentum" der Grimmnachfolger (Hübner 1930: 76) sprechen für sich.

Das neue Thesauruskonzept der inneren Vollständigkeit führte mit seinen Uneinheitlichkeiten zu Benutzungsproblemen. Es bewirkte zudem eine massive Ausdehnung der Umfangsvorstellungen. Anstelle der ursprünglich geplanten ca. 8 Bände, rechnete R. Hildebrand so schon mit ca. 15 Bänden (Meißner 1910: 78). Darüber hinaus ergab sich aus diesem Konzept eine erhebliche Verzögerung der Bearbeitungsgeschwindigkeit. Das Zusammenwirken dieser Faktoren zog mittelfristig eine krisenhafte Entwicklung des Deutschen Wörterbuchs nach sich, wie schon von Zeitgenossen vermerkt wurde (Meißner 
1910, Hübner 1933), wozu besonders auf die hier einbezogenen Arbeiten von $G$. Roethe und $A$. Hübner verwiesen sei.

Weniger beachtet vollzog sich unabhängig von der lexikographischorganisatorischen Gestaltung des Grimmschen Wörterbuchs als Nationalthesaurus eine Auseinandersetzung um seine exklusive Rolle als das eine vollständige deutsche Wörterbuch. Mit der Differenzierung des germanistischen Forschungsspektrums entstanden seit der Mitte des 19. Jahrhunderts Interessen an lexikographischer Information, die das Grimmsche Wörterbuch mit seinem Objektbereich und seiner philologischen Ausrichtung nicht berücksichtigen konnte. Exemplarisch sei hier auf die Bereiche. der Mundartlexik sowie die Fach- und Sondersprachen usw. verwiesen. Zudem mußte die stark kulturund begriffsgeschichtliche Ausrichtung des Grimmschen Wörterbuchs zwangsläufig sprachstrukturelle oder historisch-strukturelle Gesichtspunkte vernachlässigen, wie $H$. Paul in seiner Kritik zeigte (Paul 1894).

Das Konzept des einen vollständigen Nationalwörterbuchs erwies sich wissenschaftlich als nicht mehr tragfähig. Aus den Anforderungen verschiedener, vor allem wissenschaftlicher Disziplinen und Interessen entstand daher mit der Zeit eine differenzierte Wörterbuchlandschaft, die im wesentlichen der von G. W. Leibniz entworfenen Utopie entsprach. Es ist hervorzuheben, daß die neue Wörterbuchlandschaft vor allem in Auseinandersetzung mit dem Grimmschen Wörterbuch und komplementar zu dessen Objektbereich entstand. Dadurch wurde dessen Position ganz unabhängig von der skizzierten wissenschaftspolitschen Rolle eher gestützt als in Frage gestellt. Der Begriff der inneren Vollständigkeit ließ sich zwar prinzipiell weiter anwenden, jedoch nur auf den Bereich, den das Grimmsche Wörterbuch tatsächlich auch lexikographisch abdeckte, und dies war schwerpunktmäßig die deutsche National- oder Verkehrssprache ab 1450. Die hymnischen Umschreibungen der Hildebrandschen Partien im Grimmschen Wörterbuch mit Begriffen wie "köstliches Gestein" (Grimm 1893: 1431) oder "schmucken Kapellen" im "gotischen Dom" des Gesamtwerks (Pretzel 1981: 221) sprechen dafür, daß sie die Erwartungen eines Teils der Zeitgenossen an ein Wörterbuch übertrafen. Sie können jedoch nicht darüber täuschen, daß das in ihnen enthaltene Thesauruskonzept mit den wissenschaftlichen Interessen eines größeren Teils der Benutzer nicht mehr im Einklang standen. Das ursprüngliche Konzept war durch die lange Laufzeit des Unternehmens wissenschaftsgeschichtlich überholt worden.

Vor diesem Hintergrund ist der Umbruch des Hildebrandschen Thesauruskonzepts des Grimmschen Wörterbuch zu sehen, der mit dem Entstehen von Plänen eingeleitet wird, die einen Thesaurus der deutschen Sprache gegen das Deutsche Wörterbuch oder unabhängig von diesem zum Gegenstand haben (Schröter 1987). Bei diesen Plänen für einen Gegenthesaurus handelte es sich um den Versuch, in Nachahmung des 1893 gegründeten Thesaurus Linguae Latinae einen Thesaurus Linguae Germanicae aufzubauen. Dabei geht es einerseits um die Anlage eines umfassenden Großarchivs, andererseits 
um die Ausarbeitung eines darauf gestützten "vollständigen" Wörterbuchs. Die Initiative zu einem solchen Unternehmen scheint von $\mathrm{H}$. Grimm ausgegangen zu sein (Grimm 1893). Dessen knappe Ausführungen zur Gestaltung des Vorhabens lassen jedoch rasch erkennen, daß den beiden Thesauri kaum mehr als der Name gemeinsam war. So sprach $\mathrm{H}$. Grimm zwar von der "Herstellung eines Schatzes aller Deutschen Worte, ebenfalls von den Anfangen der Sprache bis auf heute" (Grimm 1893: 1430), führte jedoch dann Vorschläge ein, die eher auf ein literarisch-stilgeschichtliches Konzept schließen lassen als auf ein das gesamte Diasystem umfassendes Gesamtwörterbuch (Grimm 1893: 1431). Die Unschärfe dieser Thesaurusvorstellung blieb auch kennzeichnend für die weitere Diskussion, so daß es kaum gerechtfertigt scheint, von einem bestimmten Thesaurusplan zu sprechen. Teilweise wird die Thesaurusdiskussion um das Jahr 1900 als eine Bedrohung für den Fortgang des Deutschen Wörterbuchs aufgefaßt (Schröter 1987: 117, Hübner 1930: 88, 89). Eine solche Gefährdung läßt sich jedoch anhand der historischen Geschehnisabläufe kaum konkretisieren. Wie zu zeigen sein wird, war die Position des Deutschen Wörterbuchs als "Nationalschatz" zu dieser Zeit so gefestigt, daß ein Abbruch nicht ernsthaft zur Debatte stand.

Eine Eingabe K. Weinholds vom Jahr 1901 an das Reichsamt des Inneren, in der die Einrichtung eines Thesaurus Linguae Germanicae vorgeschlagen wurde, zu dem das Grimmsche Wörterbuch allenfalls als Vorarbeit zu betrachten sei (Schröter 1987: 116), fand wenig Gehör. Man war offenbar mehr daran interessiert, die Kritik an den Mangeln des Deutschen Wörterbuchs durch Reformen aufzufangen, als das Wörterbuch zugunsten eines Konkurrenzvorhabens zu vernachlässigen oder einzustellen. Im Jahr 1901 wandte sich das Reichsamt des Inneren an die Preußische Akademie und erbat Vorschläge für eine Reorganisation des Deutschen Wörterbuchs (Roethe 1913: 62). Die Umsetzung der daraufhin vorgelegten Pläne verzögerte sich jedoch. Nach dem Tod M. Heynes im Jahr 1906 war es wiederum das Reichsamt des Inneren, das die Preußische Akademie der Wissenschaften drängte, das Deutsche Wörterbuch unter die Leitung der Deutschen Kommission zu stellen (Roethe 1913: 62, Hübner 1930: 77). Mit Vollzug dieser Übernahme im Jahr 1908 war der institutionelle Rahmen für das Unternehmen verwirklicht, den sich J. Grimm erhofft hatte. Man kann damit auch die zweite Phase der Entwicklung einer Thesauruskonzeption im Grimmschen Wörterbuch als beendet betrachten. Das Unternehmen wurde fortan mit staatlicher Finanzierung in der Obhut einer wissenschaftlichen Akademie geführt. Die Kritik an der "unglückseligen Ruine" (Hübner 1937: 13), am "imposanten Torso" (Roethe 1913: 62) war die der Wissenschaftler. Sie betraf jedoch nur eine Seite des Problems und wog den wissenschaftspolitisch begründeten "höchsten Anspruch auf pietätvolle Behandlung" (Roethe 1913: 62) nicht auf. A. Hübner umriß die Situation so: "So ist also, wenn die Deutsche Kommission jetzt auf einen beschluss des Deutschen Wörterbuchs drangt, keineswegs der gedanke entscheidend, es müsse mit gewalt ein ende gemacht werden, um platz für einen neubau zu schaffen. 
(...) den ausschlag gibt vielmehr die tatsache, dass es mit unserem nationalen und wissenschaftlichen ansehen nicht vereinbar ist, wenn ein repräsentatives werk wie das Deutsche Wörterbuch noch jahrzehnte hindurch sich mühselig weiterschleppt" (Hübner 1930: 89).

Die Übernahme durch die Akademie schuf so nicht nur den für einen "Nationalschatz" gebotenen institutionellen Rahmen. Sie bildete vor allem die Voraussetzung dafür, das Unternehmen seiner Bedeutung entsprechend geordnet zum Abschluß zu bringen. Die Preußische Akademie leitete ab 1908 eine Reihe von Reformen ein, die auf eine qualitative und organisatorische Stabilisierung des Wörterbuchs hinausliefen. Das Kernstück der Reformen bildete die Einrichtung einer Zentralsammelstelle für Belegmaterial in Göttingen, die zwischen 1908 und 1914 etwa 2 Millionen Belege für die noch zu bearbeitenden Teile des Wörterbuchs exzerpierte (Lochner 1913: 78). Das in dieser Weise angelegte Archiv läßt sich ohne weiteres als verwirklichtes Teilstück eines Thesaurus-Archivs interpretieren, wie es $H$. Grimm oder $K$. Weinhold vorgeschwebt haben möchte. Damit war den Vertretern eines Konkurrenzthesaurus eines der wesentlichen Argumente genommen. Die stabilisierende Wirkung dieser Materialsammlung wurde durch eine Reihe von organisatorischen Maßnahmen unterstützt, die vor allem auf eine stärkere konzeptionelle Geschlossenheit, Umfangsbegrenzungen und Lautzeitbeschränkungen zielten. Die Ausprägung einer zentralen Wörterbuchplanung und Wörterbuchorganisation fand ihren vorläufigen Abschluß in der Gründung einer Arbeitsstelle mit hauptamtlichen Lexikographen in Berlin im Jahr 1930, der Einrichtung einer hierarchischen Redaktion der Artikel und der Abfassung von Arbeitsrichtlinien (Hübner 1935: 37-41). Diese Arbeitsrichtlinien machen deutlich, daß man sich auch auf die veränderte Entwicklung der deutschen Wörterbuchlandschaft einstellte. Die Bearbeiter wurden so darauf hingewiesen, daß Mundartliches ebenso wie Fach- und Sondersprachen nur sehr zurückhaltend zu behandeln waren. Auch für die Ausbreitung kulturgeschichtlicher Zusammenhänge galt deutliche Beschränkung (Richtlinien 1932: 6-12). Wenngleich viele Artikel aus der Zeit nach 1930 solche Beschränkungen ignorierten, muß doch festgestellt werden, daß in der Konzeption ein Vollständigkeitsanspruch nur noch in bezug auf die Schriftsprache besteht.

Das Prinzip der inneren Vollständigkeit behielt seine Gültigkeit auch im nunmehr modifizierten Totalitätsanspruch und bestimmte die Wörterbucharbeit bis zum Abschluß der ersten Ausgabe im Jahr 1960. Der breite Spielraum wissenschaftlichen Ermessens bei der Gestaltung der Artikel, auch im Hinblick auf das, was als Vollständigkeit gelten sollte, war eines der Probleme, die durch die Reformen zwischen 1908 und 1930 nur teilweise behoben wurden, so daß es unredlich schiene, die nur geringe Beschleunigung des Bearbeitungsablaufs während der Zeit der Akademieträgerschaft vor allem mit den Unterbrechungen durch Kriege und ungünstige äußere Umstände zu erklären. Die neu gewonnenen Materialmengen wirkten durchaus nicht nur zeitsparend, und das Programm der inneren Vollständigkeit mit dem Anspruch, einen 
monographischen Darstellungen vergleichbaren Artikel zu erstellen, erforderte auch nach den Abstrichen am Objektbereich einen ganz erheblichen Zeitaufwand.

An dieser Stelle ist noch auf die bei Benutzern bis in die Gegenwart hinein bestehende Erwartung einzugehen, mit dem Grimmschen Wörterbuch einen Thesaurus Linguae Germanicae zu benutzen. Die für die Lexikographen nach 1908 durchaus erkennbaren Veränderungen im Objektbereich des Grimmschen Wörterbuchs wurden für die Benutzer nur selten, überwiegend wohl gar nicht erkennbar. Ein klarer Umriß der Reformvorgaben nach 1908 oder 1930 wurde, soweit erkennbar ist, von den Verantwortlichen nicht an geeigneter Stelle und mit dem nötigen Nachdruck publiziert. Artikel wie GUT und GRUND lassen überdies kaum den Rückschluß zu, daß sich gegenüber dem Anspruch, das eine vollständige Wörterbuch zu sein, etwas verändert habe. Für die Neubearbeitung wird in der Einleitung zu Band I überdies durchaus von intendierter Vollständigkeit gesprochen. Insofern ist es nicht unverständlich, wenn Benutzer unkritisch einer sachlich nicht gerechtfertigten Thesaurusideologie folgen.

Der Gedanke an einen Thesaurus Linguae Germanicae im Sinne eines Gesamtwörterbuchs parallel zum Thesaurus Linguae Latinae wurde im weiteren nur noch selten vorgetragen. Th. Frings äußerte 1952 noch einmal entsprechende Pläne (Frings 1956: 328). Die Realität hatte zu diesem Zeitpunkt solche Überlegungen jedoch schon überholt. Zunächst gab es nur wenig plausible Gründe, weshalb man die in verschiedenen Wörterbüchern mit einer großen Methodenvielfalt erarbeiteten Befunde noch einmal kompilatorisch in einem Gesamtwörterbuch zusammenführen sollte. Ein solches Maximalprogramm ließ mehr Benutzungsprobleme erwarten, als es Benutzungskomfort erschloß. Ferner ergaben sich selbst aus der konzeptionell gestrafften Fortführung des Grimmschen Wörterbuchs nach 1908 bzw. 1930 gewichtige Gründe gegen Wörterbücher mit noch weitergehenden Vollständigkeitsvorgaben. Wenn schon die Bearbeitung von ca. 2 Millionen Belegen letztlich auf eine Laufzeit von fünfzig Jahren führte, welche Laufzeiten oder Personalkapazitäten waren dann für eine vollständige Erfassung und lexikographische Beschreibung des Deutschen anzusetzen? G. Roethe hatte schon 1913 in Kenntnis dieser Probleme geäußert, daß nur Fanatiker von einem solchen Werk träumen konnten (Roethe 1913: 72). An dieser nüchternen Beurteilung sind auch heute, nach sechzig Jahren, keine Abstriche vorzunehmen.

Vorschläge, ein Archiv oder ein Wörterbuch zu beginnen, in denen eine totale Erfassung des Deutschen oder auch nur aller literarischen Texte im engeren Sinn angestrebt wurde, müssen als wörterbuchgeschichtlich anachronistisch und sachfremd bezeichnet werden. Neben solchen Plänen nimmt sich selbst die einhundertzweiundzwanzigiährige Laufzeit des Grimmschen Wörterbuchs in seiner ersten Auflage aus wie ein solider Zweckbau neben dem Babylonischen Turm.

Vollständigkeit im Wörterbuch nach Art des Hildebrandschen Thesauruskonzepts hebeln die Leistungsfähigkeit der Textsorte Wörterbuch aus. Wörter- 
bücher, die für sehr umfangreiche, komplexe Objektbereiche wie die neuhochdeutsche Verkehrssprache angelegt werden, sind nach ihrer Textsortenspezifik Hilfsmittel, die einen überblickartigen Aufschluß über verschiedene Bereiche des Wortschatzes und der einzelnen Wörter geben können. Sie ersetzen weder die wissenschaftlichen Monographien zu den Einzelwörtern noch Wortfeldoder Wortfamilienanalysen. Wenn solche Wörterbuchinformation für den Benutzer wirklich eine Hilfestellung sein soll, muß sie in sich abgeschlossen und übersichtlich organisiert sein. Sie muß für wissenschaftliche Ansprüche vor allem mit dem aktuellen Forschungsstand korrespondieren. Der zuletzt erwähnte Gesichtspunkt verlangt, daß die Gesamtbearbeitung einen überschaubaren Zeitraum nicht überschreitet. Bei der heute üblichen Entwicklung auch in den Geisteswissenschaften wäre daher ein Laufzeitmaximum von dreiBig Jahren anzusetzen. Vollständigkeit im Sinn des Hildebrandschen Thesauruskonzepts von der inneren Vollständigkeit wird zum kontraproduktiven Faktor, wenn sie nicht durch eine streng auf Laufzeit und Umfang bezogene Konzeption begrenzt wird. Im internationalen Vergleich, so zeigt die Entwicklung der Großwörterbuchplanung seit 1945, hat sich das Konzept des Oxford English Dictionary durchgesetzt und nicht das des Grimmschen Wörterbuchs.

Mit Blick darauf ist festzustellen, daß bei der Gründung der Neubearbeitung des Grimmschen Wörterbuchs nicht genügend Lehren aus der Unternehmensgeschichte gezogen worden sind. Der Plan, die am stärksten veralteten Teile des Grimmschen Wörterbuchs im Buchstabenbereich A-F durch eine völlige Neubearbeitung zu ersetzen, orientierte sich bei den Umfangsplanungen zur Festlegung der Materialbasis und des Darstellungsbereichs an der jüngeren Praxis der abgeschlossenen Ausgabe. Die Formulierung man schließe sich den besten Traditionen der alten Ausgabe an, bedeutete die Fortsetzung des Bemühens um eine wissenschaftlich vollständige Darstellung der Einzelwortgeschichten für die Schriftsprache (Neumann 1958). Gegenentwürfe von J. Erben für die Neubearbeitung in Form eines mehrbändigen, komprimierten Wörterbuchs (Erben 1957) konnten sich gegen die Fortführung des Thesauruskonzepts von Th. Frings und $\mathrm{H}$. Neumann nicht durchsetzen. Die mit diesem Konzept schon für die abgeschlossene Ausgabe verbundenen Probleme wiederholten sich zwangsläufig bei der Neubearbeitung, so daß Mitte der achtziger Jahre nach einer Laufzeit von 25 Jahren eine weitreichende Unternehmensreform erforderlich wurde. Die damit zusammenhängenden Veränderungen werden hier dem vierten Abschnitt der Entwicklung des Thesauruskonzepts zugeordnet.

Neben der notwendigen Beschleunigung der laufenden Bearbeitung hat es sich die Reform der Neubearbeitung auch zur Aufgabe gemacht, die in der Unternehmensphilosophie angelegten Thesauruskomponenten in einen tragfähigen Zusammenhang einzubetten.

Die unternehmensgeschichtlichen Beobachtungen zeigen, daß die wissenschaftlichen Vollständigkeitsvorstellungen für ein Wörterbuch zum Problem werden können, wenn ihre Verwirklichung zu einer sehr langen Bearbeitungs- 
zeit führt. Durch die Entfernung der Wörterbuchkonzeption von der Entwicklung der Forschungsinteressen kann ein Akzeptanzverlust eintreten. Kürzungen des Umfangs und Vereinfachung der Bearbeitung zur Beschleunigung konnten vordergründig als einfache, aber wirksame Lösungen des Problems betrachtet werden. Gegen solche Lösungen spricht, daß das Grimmsche Wörterbuch seit Beginn des Jahrhunderts eine spezifische Rolle als umfassendes historisches Großwörterbuch der deutschen Schriftsprache in einer arbeitsteiligen Wörterbuchlandschaft einnimmt und dementsprechend auch umfassende Information zum Wortbestand und Wortgebrauch der neuhochdeutschen Wörter ab 1450 anbieten muß. Diese Informationsdifferenzierung ergibt sich auch aus der verbreiteten Betrachtung des Grimmschen Wörterbuchs als Gesamtwörterbuch der Leitvarietät des Deutschen, analog zu anderen europäischen Wörterbüchern mit nationalsprachlicher Ausrichtung. Die Forderung nach Abgeschlossenheit und Überschaubarkeit der Wörterbuchinformation ist jedoch mit dem gleichzeitigen Bemühen um Erschließung möglichst vieler Detailinformationen in einem Medium kaum angemessen zu vermitteln. Das Reformkonzept sieht daher eine Aufgabenverteilung vor, bei der das gedruckte Wörterbuch seiner Hilfsmittelfunktion unter Einhaltung vertretbarer Laufzeiten gerecht werden kann, während die Fülle der Detailinformationen in ein Thesaurus-Archiv verlagert wird.

Das Wörterbuch soll nicht mehr Vollständigkeit im monographischen Verständnis anstreben, sondern sich in der Darstellung auf die historisch wesentlichen und usuellen wortgeschichtlichen Sachverhalte beschränken. Damit tritt das ursprüngliche Konzept der gewichteten Fülle wieder in den Blick. Es zeigt nicht mehr die Handschrift des Sprachmeisters, der richtige und vorbildliche Sprachverwendungen auswählt oder den besonderen poetischen Inhalt vermitteln will. Ziel ist es vielmehr, einen sprachgeschichtlich deskriptiven, anschaulichen Querschnitt durch den Bestand der Stichwörter und ihre Verwendungen zu gewährleisten. Die Breite der Darstellung ist einerseits durch die Laufzeit und das vorgegebene Bandvolumen bestimmt, andererseits aber vor allem durch den Bestand an konventionellen, sprachgeschichtlich erkennbar bezeugten Vorkommen.

Mit dieser Ausrichtung am Prinzip der gewichteten Fülle läßt sich gegenüber dem Bemühen um innere Vollständigkeit eine nennenswerte Beschleunigung der laufenden Neubearbeitung erreichen, ohne daß die Aufgabenstellung des Deutschen Wörterbuchs als die maßgebliche Gesamtdarstellung der neuhochdeutschen Leitvarietät nennenswert berührt wurde. Ein Abschluß des Unternehmens in überschaubarer Zeit fordert den Preis, sich im Verständnis des oben zitierten Benutzers auf lexikographische "Stangenware" einzulassen. Kritikern ist entgegenzuhalten, daß in jedem Fall ein abgeschlossenes Wörterbuch auch minderen Umfangs sinnvoller benutzbar ist als der umfänglichste Thesaurus-Torso.

Man kommt jedoch nicht umhin festzustellen, daß eine Reihe von Benutzerwünschen mit diesem Wörterbuchkonzept nicht zu befriedigen ist. 
Dazu ist vor allem der Wunsch nach umfänglichem Belegmaterial und Nachweis auch seltener, besonderer Vorkommen zu rechnen. Im Rahmen der genannten Aufgabenteilung sieht das Reformkonzept hier den Aufbau eines Großarchivs vor, das auch für solche weitergehenden Interessen Nutzungszugriffe erschließen soll. Dieses Großarchiv wird in Form einer Textdatenbank und einer darauf gestützten Wortdatenbank mit angeschlossenem Belegarchiv auf elektronischer Basis angelegt. Das neue Wortarchiv soll erstmalig in der Unternehmensgeschichte des Grimmschen Wörterbuchs Belegmaterial für den gesamten Wortbestand von A-Z in einem sehr großen Volumen erschließen. Erste Zugriffsmöglichkeiten können ab 1995 erwartet werden. Eine Anlage des Wortarchivs als strukturierte Datenbank erlaubt programmgestützt eine Reihe von Informationen zu jedem Wort zu geben, die bei der Anlage von Zettelarchiven nur mit sehr großem Arbeitsaufwand denkbar waren wie Bezeugungsdauer, Belegfrequenz usw. Damit nähert sich diese Datenbank zugleich dem Typ des Wissensthesaurus, wie ihn die heutige Informationstechnik anstrebt. Durch die Stützung auf ein flexibles Texterfassungsmodell kann das Wortschatzarchiv grundsätzlich offengehalten werden. Die Menge und Schichtung der berücksichtigten Texte kann daher nach Forschungsinteressen und Benutzerwünschen auch längerfristig modifiziert werden. Damit entsteht auf Dauer ein Wortschatzarchiv, das eine zeitgemäße Entsprechung zu dem um die Jahrhundertwende parallel zum Thesaurus Linguae Latinae geplanten Großarchiv darstellt. Ohne Vollständigkeitsansprüche zu stellen, kann ein solches Archiv zum zentralen Dokumentationsmittel für die neuhochdeutsche Verkehrssprache werden. Ebenso schafft es die Grundlage für eine mögliche Fortführung der Neubearbeitung des Grimmschen Wörterbuchs.

\section{Literaturhinweise}

Deutsches Wörterbuch. 1854. Deutsches Wörterbuch von Jacob Grimm und Wilhelm Grimm. Erster Band. 1854 ff. Leipzig: S. Hirzel Verlag.

Dückert, J. (Red.). 1987. Das Grimmsche Wörterbuch. Untersuchungen zur lexikographischen Methodologie. Leipzig: S. Hirzel Verlag.

Dückert, J. 1987. Jacob Grimm und Wilhelm Grimm. Dückert, J. (Red.). 1987: 7-48.

Erben, J. 1957. [Bericht über einen Vorschlag zur Erarbeitung eines 'mittleren Grimm' in der Sitzung der Deutschen Kommission vom 3. 4. 1957]. Maschinenschriftl. Exemplar. Archiv Arbeitstelle Göttingen.

Frings, Th. 1956. Aufgaben und Ziele des Instituts für deutsche Sprache und Literatur. Wiederabdruck einer 1952 bei der Eröffnung des Instituts gehaltenen Ansprache, Deutsche Akademie der Wissenschaften zu Berlin 1946-1956: 320-329.

Georges, KE. (Red.). 1951. Ausführliches Lateinisch-Deutsches Handwörterbuch. Leipzig: Hahnsche Buchhandlung.

Grimm, H. 1893. Thesaurus linguae germanicae. Deutsche Litteraturzeitung. Nr. 45, 11. November 1893: 1430-1432. 
Hildebrand, R. 1869. Über Grimms Wörterbuch in seiner wissenschaftlichen und nationalen Bedeutung. Leipzig: S. Hirzel Verlag.

Hildebrand, R. 1890. Gesammelte Aufsätze und Vorträge zur deutschen Philologie und zum deutschen Unterricht. Leipzig: Teubner Verlag.

Hübner, A. 1930. Die Lage des Deutschen Wörterbuches. Anzeiger fïr deutsches Altertum 49: 73-90.

Hübner, A. 1933. Die Lage des Deutschen Wörterbuches. Forschungen und Fortschritte 9: 101-102.

Hübner, A. 1935. Festvortrag in der Preußischen Akademie der Wissenschaften am 4. 1. 1935. Kunisch, H. und U. Pretzel (Red.). 1940: 26.

Hübner, A. 1937. Gustav Roethe als wissenschaftlicher Organisator. Rede beim Grimm-Fest der Gesellschaft für deutsche Philologie, am 9. Januar 1937. Berlin: Privatdruck.

Kirkness, A. 1980. Geschichte des Deutschen Wörterbuchs 1883-1863. Dokumente zu den Lexikographen Grimm. Mit einem Beitrag von L. Denecke. Stuttgart: S. Hirzel Verlag.

Kunisch, H. und U. Pretzel (Red.). 1940. Arthur Hübner. Kleine Schriften zur deutschen Philologie. Berlin: Ebering.

Leibniz, G.W. 1717. Unvorgreiffliche Gedancken, betreffend die Ausübung und Verbesserung der Teutschen Sprache. Schmarsow, A. (Red.). 1877.

Lochner, J. 1913. Die Tätigkeit der Zentralsammelstelle des Deutschen Wörterbuchs seit ihrer Gründung. Neue Jahrbücher für das klassische Altertum 31: 74-81.

Meißner, R. 1910. Zur Geschichte des Grimmschen Wörterbuchs. Preußische Jahrbücher 142: 62-80.

Neumann, H. (Red.). 1958. Zur Neubearbeitung des Deutschen Wörterbuchs des Brïder Grimm. Zusammengestellt von den beiden Arbeitsstellen nach der Berliner Besprechung vom 22. 10. 1958. Den Präsidenten der deutschen Akademien vorgelegt am 8. 11. 1958 in Göttingen. Maschinenschriftl. Exemplar. Archiv Arbeitsstelle Göttingen.

Paul, H. 1894. Ueber die Aufgaben der wissenschaftlichen Lexikographie mit besonderer Rücksicht auf das deutsche Wörterbuch. Sitzungsberichte der philosophisch-philologischen und der historischen Classe der Königlich Bayerischen Akademie der Wissenschaften zu München. Heft 1: 53-91.

Pfütze, M. 1962. Jacob Grimm, das "Deutsche Wörterbuch" und die Nation - Bemerkungen zu einer politischen Entwicklung. Weimarer Beiträge 8: 264-269.

Pretzel, U. 1981. Zur Geschichte des Deutschen Wörterbuchs. Brïder Grimm Gedenken 3: 216-248.

Richtlinien. 1932. Richtlinien für die Arbeit am Deutschen Wörterbuch der Brüder Grimm. Maschinenschriftl. Exemplar. Archiv Arbeitsstelle Göttingen.

Roethe, G. 1913. Die Deutsche Kommission der Königlich Preußischen Akademie der Wissenschaften, ihre Vorgeschichte, ihre Arbeiten und Ziele. Neue Jahrbïcher für das Klassische Altertum 31: 37-74.

Sanders, D. 1852. Das deutsche Wörterbuch von Jakob Grimm und Wilhelm Grimm kritisch beleuchtet. Hamburg: Hoffmann und Campe.

Schirokauer, A. 1957. Das Grimmsche Wörterbuch als Dokument der Romantik. Philobiblon 1: 308-323.

Schmarsow, A. 1877. Leibniz und Schottelius. Die Unvorgreiflichen Gedanken, Quellen und Forschungen zur Sprach- und Culturgeschichte der Germanischen Völker XXIII. Strassburg / London.

Schröter, U. 1987. Von Moriz Heyne zur Deutschen Kommission. Zur Bearbeitung des Deutschen Wörterbuchs von 1867 bis 1908. Dückert, J. (Red.). 1987: 91-124. 
Stōtzel, G. 1970. Das Abbild des Wortschatzes. Zur lexikographischen Methode in Deutschland von 1617-1967. Poetica 3: 1-23.

Tätigkeitsbericht. 1993/94. Tätigkeitsbericht der Arbeitsstelle Göttingen des Deutschen Wörterbuchs. Göttingen. 